\title{
31 SERUM LAG-3 IS ASSOCIATED WITH IMPROVED PATIENT PROGNOSIS IN HIGH GRADE SEROUS OVARIAN CANCER
}

${ }^{1}$ Nicole James*, ${ }^{1}$ Katrin Eurich, ${ }^{1}$ Erin Lips, ${ }^{2}$ Payton De La Cruz, 'Morgan Woodman, ${ }^{1}$ Jennifer Ribeiro. 'Women and Infants Hospital, Providence, RI, USA; ${ }^{2}$ Brown University, Providence, RI, USA

Background High grade serous ovarian cancer (HGSOC) is a lethal gynecologic malignancy with a five-year survival rate of only 39 percent. ${ }^{1}$ Despite the fact that ovarian tumors are considered immunologic, HGSOC patients respond poorly to PD-1 based immunotherapy. Hence, the need to identify novel prognostic and therapeutic immunologic factors is crucial. Our previous investigation uncovered intratumoral levels of immune co-inhibitory receptor LAG-3 as a marker of improved HGSOC patient survival. For this current study we sought to evaluate the prognostic utility of serum-based LAG3 , as well as determine how these circulating levels change in response to HGSOC standard of care therapy.

Methods This study was approved by the Women and Infants Institutional Review Board; approval number 1057626. HGSOC serum samples were obtained from the Department of Special Testing and the Program in Women's Oncology Gynecologic Tissue Bank at Women and Infants Hospital. A total of 43 HGSOC treatment naïve serum samples were tested for serum LAG-3 and in 20 of these patients, samples from on- and post- frontline platinum-based chemotherapy were also analyzed. A commercially available ELISA kit was employed to detect serum LAG-3.

Results There was no statistically significant change in pre-, on-, and post- serum LAG-3 levels following frontline chemotherapy, however median levels of LAG-3 decreased once patients initiated therapy and remained stable post-treatment. Spearman Rank Correlation analysis revealed a significant relationship between progression-free survival (PFS) and pre-treatment serum LAG-3 levels $(r=0.36, p=0.017)$. Furthermore, it was found that patients with a PFS of 6 months or less exhibited a significantly $(p=0.0031)$ lower mean rank of pretreatment serum LAG-3 levels, compared to patients with a PFS of 18 months or longer. Finally, Kaplan Meier curve analysis revealed a statistically significant association between longer patient PFS and higher pre-treatment LAG-3 concentrations, when stratified by both median $(\mathrm{HR}=0.4916$, log-rank $p=0.022$ ) and quartile LAG-3 serum levels $(\mathrm{HR}=0.2679$, log-rank $\mathrm{p}=0.0031)$.

Conclusions This study demonstrates for the first time that circulating immune checkpoint receptors have prognostic capabilities in ovarian cancer. Our findings suggest that LAG-3 is a marker for improved patient PFS. Future directions include an expansion of this original cohort in order to validate and further assess the clinical prognostic utility of serum LAG-3 in HGSOC.

\section{REFERENCE}

1. Survival Rates for Ovarian Cancer, American Cancer Society, [https://www.cancer. org/cancer/ovarian-cancer/detection-diagnosis-staging/survival-rates.html]

http://dx.doi.org/10.1136/jitc-2021-SITC2021.031 